TAIWANESE JOURNAL OF MATHEMATICS

Vol. 10, No. 4, pp. 1069-1082, June 2006

This paper is available online at http://www.math.nthu.edu.tw/tjm/

\title{
TOPOLOGY INDUCED BY QUOTIENTS ON NEARRING MODULES
}

\author{
Feng-Kuo Huang
}

\begin{abstract}
The aim of this paper is to study one special quotient in the class of nearring modules. Contrast to the well known noetherian quotient, this quotient is less noticed. Results and applications for this quotient are developed and an induced topology in the class of type- $1 N$-modules is introduced.
\end{abstract}

\section{INTRODUCTION}

Let $G$ and $N$ be two algebraic objects with $N$ acting on $G$ from the right hand side. This is usually denoted as $G \times N \rightarrow G$ with $a n=b$ for $a, b \in G$ and $n \in N$. Two linear equations, say $a x=b$ for given $a, b \in G$ and $y n=b$ for given $n \in N, b \in G$, can be informally imposed. To solve the equation $a x=b$ on $N$, the noetherian quotient $(b: a)$ is introduced to denote the solution set in $N$. As the other equation $y n=b$, the quotient $[b: n]$ will be used to denote the solution set in $G$ [7, p. 41].

Let $(G,+)$ be a group (not necessarily abelian) and $(N,+, \cdot)$ a left nearring. We call $G$ a (right) $N$-module if $a(f+g)=a f+a g$ and $a(f g)=(a f) g$ for all $a \in G$ and all $f, g \in N$. Given subsets $X, Y \subseteq G$ and $U \subseteq N$. Two quotients can be defined as following:

$$
\begin{aligned}
& (X: Y):=\{f \in N \mid a f \in X \text { for all } a \in Y\} \text { and } \\
& {[X: U]:=\{a \in G \mid a f \in X \text { for all } f \in U\} .}
\end{aligned}
$$

The first one is the usual noetherian quotient commonly used in the class of ring modules. The other while less studied is first defined in [7, p. 41] and will be called in this paper as the inverse quotient of $X$ by $U$. When $X=\{0\}$, we write

Received December 8, 2004, revised March 28, 2005.

Communicated by Shun-Jen Cheng.

2000 Mathematics Subject Classification: Primary 16Y30; Secondary 16 Y99.

Key words and phrases: Nearring module, Quotient, Topology.

The author was partially supported by the National Science Council under the grant number NSC 92-2115-M-143-001. 
$(0: Y)$ and $[0: U]$ for $(\{0\}: Y)$ and $[\{0\}: U]$ respectively. The set $(0: Y)$ is the annihilator of $Y$ in $N$ and is sometimes denoted as $\operatorname{Ann}(Y)$ or $\operatorname{Ann}_{N}(Y)$ in literatures. The set $[0: U]$ is denoted as $Z(U)$ and called the zero set of $U$ in $G$ by Scott [10]. In fact, it is called an affine algebraic variety if we consider the polynomial ring $F\left[x_{1}, x_{2}, \cdots, x_{n}\right]$ acting on $F^{n}$ where $F$ is an algebraicly closed fields. Note that the set $[0: U]$ had been used by Johnson $[4,5]$ to characterize the maximal right ideals in the class of transformation nearrings.

A topology had been introduced by Scott in [10] using zero sets $[0: U]$ for 2primitive nearrings and further studied the applications of this topology in the class of primary $N$-modules [11]. The attemp of this paper is to introduce a topology using inverse quotient in the class of $N$-modules. It is shown in this paper that: Given $N$ a 1-primitive nearring on a group $G$. If $G$ is a nonabelian group or $N_{0}$ a nonring, then the collection of sets $G \backslash[0: U]$ for all $U \subseteq N$ forms a topology in $G$ (by Corollary 2.2 and Corollary 3.4), and all the mappings of $G$ induced by elements in $N$ are continuous with respect to this topology (Theorem 3.8). Examples are given to demonstrate and delimit our results. For other terminologies on nearring theory not defined in this paper, please refer to [7, 9] but note that [9] using right nearrings instead of left nearrings. Terminologies on topology are referred to Munkres [8].

\section{INVERSE QUOTIENT}

Let $G$ be a (right) $N$-module. If $X$ is a subset of $G$ and $U$ is a subset of $N$, the following convention will be made for the inverse quotient of $X$ by $U$ throughout this paper. If $U=\{0\}$, we write $[X: 0]$ in place of $[X:\{0\}]$. A similar simplification will be used if $X$ or $U$ is a singleton. When $X$ or $U$ is an empty set, we make the following convention that $[X: \emptyset]=G ;[\emptyset: U]=\emptyset$ and leave $[\emptyset: \emptyset]$ undefined.

Proposition 2.1. Assume $G$ is an $N$-module. Let $X, U$ be subsets of $G$ and $N$ respectively. Suppose $\left\{X_{i}\right\}_{i \in I}$ is a collection of subsets of $G$ and $\left\{U_{j}\right\}_{j \in J}$ is a collection of subsets of $N$. Then we have:

(1) $\bigcap_{j \in J}\left[X: U_{j}\right]=\left[X: \bigcup_{j \in J} U_{j}\right]$. In particular, $[X: U]=\bigcap_{f \in U}[X: f]$.

(2) $\bigcup_{j \in J}\left[X: U_{j}\right] \subseteq\left[X: \bigcap_{j \in J} U_{j}\right]$. If $\left\{U_{j}\right\}_{j \in J}$ is a chain by using set inclusion, then $\bigcup_{j \in J}\left[X: U_{j}\right]=\left[X: \bigcap_{j \in J} U_{j}\right]$.

(3) $\bigcap_{i \in I}\left[X_{i}: U\right]=\left[\bigcap_{i \in I} X_{i}: U\right]$.

(4) $\bigcup_{i \in I}\left[X_{i}: U\right] \subseteq\left[\bigcup_{i \in I} X_{i}: U\right]$. If $\left\{X_{i}\right\}_{i \in I}$ is a chain by using set inclusion, then $\bigcup_{i \in I}\left[X_{i}: U\right]=\left[\bigcup_{i \in I} X_{i}: U\right]$. 
Proof. (1) Observe that

$$
\begin{aligned}
{\left[X: \bigcup_{j \in J} U_{j}\right] } & =\left\{a \in G \mid a f \in X \text { for all } f \in \bigcup_{j \in J} U_{j}\right\} \\
& =\bigcap_{j \in J}\left\{a \in G \mid a f \in X \text { for all } f \in U_{j}\right\}=\bigcap_{j \in J}\left[X: U_{j}\right] .
\end{aligned}
$$

(2) If $\bigcap_{j \in J} U_{j}=\emptyset$, then it is clear that

$$
G=\left[X: \bigcap_{j \in J} U_{j}\right] \supseteq \bigcup_{j \in J}\left[X: U_{j}\right] .
$$

If $\bigcap_{j \in J} U_{j} \neq \emptyset$, then

$$
\begin{aligned}
\bigcup_{j \in J}\left[X: U_{j}\right] & =\bigcup_{j \in J}\left\{a \in G \mid a f \in X \text { for all } f \in U_{j}\right\} \\
& \subseteq\left\{a \in G \mid a f \in X \text { for all } f \in \bigcap_{j \in J} U_{j}\right\}=\left[X: \bigcap_{j \in J} U_{j}\right] .
\end{aligned}
$$

It is clear that if $\left\{U_{j}\right\}_{j \in J}$ is a chain then the above inclusion becomes equality.

(3) If $\bigcap_{i \in I} X_{i}=\emptyset$, it is clear by definition that our assertion holds. We now suppose $\bigcap_{i \in I} X_{i} \neq \emptyset$.

$$
\begin{aligned}
{\left[\bigcap_{i \in I} X_{i}: U\right] } & =\left\{a \in G \mid a f \in \bigcap_{i \in I} X_{i} \text { for all } f \in U\right\} \\
& =\bigcap_{i \in I}\left\{a \in G \mid a f \in X_{i} \text { for all } f \in U\right\}=\bigcap_{i \in I}\left[X_{i}: U\right]
\end{aligned}
$$

(4) Observe that

$$
\begin{aligned}
\bigcup_{i \in I}\left[X_{i}: U\right] & =\bigcup_{i \in I}\left\{a \in G \mid a f \in X_{i} \text { for all } f \in U\right\} \\
& \subseteq\left\{a \in G \mid a f \in \bigcup_{i \in I} X_{i} \text { for all } f \in U\right\}=\left[\bigcup_{i \in I} X_{i}: U\right] .
\end{aligned}
$$

It is clear that if $\left\{X_{i}\right\}_{i \in I}$ is a chain then the above inclusion becomes equality.

Let $X$ be a fixed subset of $G$. A subset $Y$ of $G$ will be called $X$-closed if there exists a subset $U$ of $N$ such that $[X: U]=Y$. Since $G=[X: \emptyset]$, the group 
$G$ is considered as an $X$-closed set for all specified nonempty set $X$. Hereafter, the empty subset $\emptyset$ of $G$ shall also be regarded as an $X$-closed set. The following results are immediate from Proposition 2.1.

Corollary 2.2. Any intersection of $X$-closed subsets of an $N$-module $G$ is $X$-closed.

Corollary 2.3. Let $U_{1}, U_{2}$ be subsets of $N$ and $X$ a subset of $G$. If $U_{1} \subseteq U_{2}$, then $\left[X: U_{1}\right] \supseteq\left[X: U_{2}\right]$.

Proposition 2.4. Let $X, Y$ be nonempty subsets of an $N$-module $G$. Then $Y \subseteq[X:(X: Y)]$. Furthermore, $Y=[X:(X: Y)]$ if and only if $Y$ is $X$-closed.

Proof. If $(X: Y)$ is empty, then it is clear that $Y \subseteq[X:(X: Y)]=G$. If $(X: Y)$ is not empty, then $Y(X: Y) \subseteq X$ and so $Y \subseteq[X:(X: Y)]$.

Suppose $Y=[X:(X: Y)]$, then $Y$ is clearly $X$-closed. On the other hand, suppose that $Y$ is $X$-closed, say $Y=[X: U]$ for some subset $U$ of $N$. If $U$ is empty then $Y=G$, so we have $[X:(X: Y)] \subseteq Y$. If $U$ is not empty, then $Y U \subseteq X$ and so $U \subseteq(X: Y)$. Corollary 2.3 then implies that $[X:(X: Y)] \subseteq[X: U]=Y$. Hence $[X:(X: Y)]=Y$.

Corollary 2.5. Let $X, W$ be subsets of an $N$-module $G$. Suppose $Y$ is an $X$-closed subset of $G$. Then we have $(X: Y) \subseteq(X: W)$ if and only if $W \subseteq Y$.

Proof. The necessary part is trivial. We need to show that it is sufficient. From Corollary 2.3, it follows that $[X:(X: W)] \subseteq[X:(X: Y)]$. Therefore $W \subseteq[X:(X: W)] \subseteq[X:(X: Y)]=Y$ by Proposition 2.4.

The assumption that $Y$ is $X$-closed in $G$ in Corollary 2.5 is not superfluous. For instance, let $G=\left(\mathbb{Z}_{3},+\right)=(\{0,1,2\},+)$ be the group of order three written additively and $N=M_{c}(G)=\left\{\theta_{0}, \theta_{1}, \theta_{2}\right\}$ the nearring of constant mappings on $G$. Let $X=\{0\}, Y=\{1\}, W=\{2\}$ be subsets of $\mathbb{Z}_{3}$. Observe that $\mathbb{Z}_{3}$ and $\emptyset$ are the only 0 -closed sets, and thus $Y$ is not $X$-closed in this case. However, $(X: Y)=(0: 1)=\left\{\theta_{0}\right\} \subseteq(X: W)=(0: 2)=\left\{\theta_{0}\right\}$ but $\{2\} \not\{\{1\}$.

Corollary 2.6. Let $X$ be a subset of an $N$-module $G$. Suppose $Y_{1}$ and $Y_{2}$ are $X$-closed subsets of $G$. Then $Y_{1}=Y_{2}$ if and only if $\left(X: Y_{1}\right)=\left(X: Y_{2}\right)$.

Proof. This follows immediately from Corollary 2.5.

Corollary 2.6 reveals that there is a one-one correspondence between $X$-closed subsets of $G$ and their noetherian quotient with respect to $X$. This correspondence is lattice reversing as indicated in Corollary 2.5. 
It is also interesting to see that most of the conclusions above have "dual" results when the role of noetherian quotient and inverse quotient interchanged. We put some of them in the following proposition.

Proposition 2.7. Let $X, Y_{1}, Y_{2}$ be nonempty subsets of an $N$-module $G$ and $U, U_{1}, U_{2}$ nonempty subsets of $N$.

(1) If $Y_{1} \subseteq Y_{2}$, then $\left(X: Y_{1}\right) \supseteq\left(X: Y_{2}\right)$.

(2) The set $U \subseteq(X:[X: U])$. Furthermore, $U=(X:[X: U])$ if and only if $U=(X: Y)$ for some $Y \subseteq G$.

(3) Assume $U_{1}=(X: Y)$ for some $Y \subseteq G$. Then $\left[X: U_{1}\right] \subseteq\left[X: U_{2}\right]$ if and only if $U_{2} \subseteq U_{1}$.

(4) Suppose $U_{1}=\left(X: Y_{1}\right)$ and $U_{2}=\left(X: Y_{2}\right)$. Then $U_{1}=U_{2}$ if and only if $\left[X: U_{1}\right]=\left[X: U_{2}\right]$

\section{Proof.}

(1) This result follows from the identity that $\cap_{j \in J}\left(X: Y_{j}\right)=\left(X: \cup_{j \in J} Y_{j}\right)$.

(2) It suffices to show the case when $[X: U] \neq \emptyset$. Observe that $[X: U] U \subseteq X$, and thus $U \subseteq(X:[X: U])$.

Furtheremore, if $U=(X:[X: U])$, then simply let $Y=[X: U] \subseteq G$. On the other hand, assume $U=(X: Y)$ for some $Y \subseteq G$. If $Y=\emptyset$, then $U=N$ and thus $(X:[X: U]) \subseteq U$. If $Y \neq \emptyset$, then $Y U \subseteq X$ and so $Y$ $\subseteq[X: U]$. Therefore $(X:[X: U]) \subseteq(X: Y)=U$ by (1). Hence result.

(3) Assume $\left[X: U_{1}\right] \subseteq\left[X: U_{2}\right]$ where $U_{1}=(X: Y)$ for some $Y \subseteq G$. Observe that $\left(X:\left[X: U_{1}\right]\right) \supseteq\left(X:\left[X: U_{2}\right]\right)$ by (1) and $U_{2} \subseteq\left(X:\left[X: U_{2}\right]\right)$ by (2). Therefore $U_{2} \subseteq\left(X:\left[X: U_{2}\right]\right) \subseteq\left(X:\left[X: U_{1}\right]\right)=U_{1}$ by (2) again.

On the other hand, assume $U_{2} \subseteq U_{1}$. Let $a \in\left[X: U_{1}\right]$. Then $a \mu \in X$ for all $\mu \in U_{1}$. Consequently $a \mu \in X$ for all $\mu \in U_{2}$ or $a \in\left[X: U_{2}\right]$. Therefore $\left[X: U_{1}\right] \subseteq\left[X: U_{2}\right]$.

(4) This result follows immediately from (3).

Proposition 2.8. Let $X, Y$ be subsets of an $N$-module $G$ and $f \in N$. If $Y$ is $X$-closed, then $[Y: f]=[X: f(X: Y)]$.

Proof. If $[Y: f]=\emptyset$, then clearly $[Y: f] \subseteq[X: f(X: Y)]$. If $(X: Y)=\emptyset$, then $[X: f(X: Y)]=G$ and so $[Y: f] \subseteq[X: f(X: Y)]$. Suppose that both $[Y: f] \neq \emptyset$ and $(X: Y) \neq \emptyset$. Since $[Y: f] f \subseteq Y$, we have $[Y: f] f(X: Y) \subseteq$ $Y(X: Y) \subseteq X$. Therefore $[Y: f] \subseteq[X: f(X: Y)]$. 
On the other hand, if $[X: f(X: Y)]=\emptyset$, then clearly $[X: f(X: Y)] \subseteq$ $[Y: f]$. Suppose that $[X: f(X: Y)] \neq \emptyset$ and $v \in[X: f(X: Y)]$. Then $v f(X: Y) \in X$ and so $(X: Y) \subseteq(X: v f)$. Thus $v f \in Y$ by Corollary 2.5 and hence $v \in[Y: f]$ or $[X: f(X: Y)] \subseteq[Y: f]$. Hence result.

Let $N$ be a nearring, $G$ an $N$-module. A subgroup $H$ of $G$ such that $a f \in H$ for all $a \in H, f \in N$ is called an $N$-submodule of $G$. A nearring $N$ is called a distributively generated nearring (abbre. $d$. g. nearring) if $(N,+)$ is generated as a group by a semigroup $(S, \cdot)$ of distributive elements in $N$. This d. g. nearring is generally denoted $(N, S)$ or $N[S]$. An $N$-module $G$ is called a $d$. g. module or $N[S]$-module provided that $N[S]$ is a d. g. nearring and that $(a+b) s=a s+b s$ for all $a, b \in G$ and all $s \in S$. In general, there is no particular algebraic structure for inverse quotient in an $N$-module. However, some can be said in an $N[S]$-module.

Proposition 2.9. Suppose $G$ is an $N[S]$-module. Let $K$ be a subset of $G$ and $U$ a subset of $S$.

(1) If $K$ is a subgroup of $G$ and $f \in S$, then $[K: f]$ is a subgroup of $G$.

(2) If $K$ is a subgroup of $G$, then $[K: U]$ is a subgroup of $G$.

(3) If $K$ is an $N$-submodule of $G$ and $U$ is contained in the multiplicative center of the semigroup $(S, \cdot)$, then $[K: U]$ is an $N$-submodule of $G$.

(4) If $K$ is a subgroup of $G$ and $S U \subseteq U$, then $[K: U]$ is an $N$-submodule of $G$. In particular, $[K: S]$ is an $N$-submodule of $G$ for any subgroup $K$ of $G$.

\section{Proof.}

(1) Since $f \in S, f$ induces an endomorphism on $G$. By corresponding theorem for groups and our assumption that $K$ is a group, it is routine to see that $[K: f]$ is a subgroup of $G$.

(2) From Proposition 2.1 and (1) above.

(3) From (2), we know that $[K: U]$ is a subgroup of $G$. We need to show that $[K: U] f \subseteq[K: U]$ for all $f \in N$. Let $a \in[K: U]$. Then $a \alpha \in K$ for all $\alpha \in U$. Now $(a f) \alpha=(a \alpha) f \in K f \subseteq K$, so af $\in[K: U]$ for all $f \in N$. Therefore $[K: U]$ is an $N$-submodule of $G$.

(4) Let $a \in[K: U], \alpha \in U$ and $f \in N$. Since $N$ is d. g., we can write $f$ as $f=\sum_{i=1}^{n} \varepsilon_{i} \alpha_{i}$ where $\varepsilon_{i} \in\{1,-1\}$ and $\alpha_{i} \in S$ for $i \in\{1,2, \cdots, n\}$. Now we have

$$
\text { (af) } \alpha=\left(a \sum_{i=1}^{n} \varepsilon_{i} \alpha_{i}\right) \alpha=\sum_{i=1}^{n} \varepsilon_{i}\left(a \alpha_{i}\right) \alpha=\sum_{i=1}^{n} \varepsilon_{i} a\left(\alpha_{i} \alpha\right) \in K .
$$

Therefore af $\in[K: U]$ and so $[K: U]$ is an $N$-submodule of $G$. 


\section{TOPOLOGy Induced by INVERSE Quotient}

The purpose of this section is to introduce a topology on an $N$-module $G$ using $X$-closed sets. Let $N$ be a nearring, $G$ an $N$-module. A normal subgroup $K$ of $G$ such that $(k+m) n-m n \in K$ for all $k \in K ; m, n \in N$ is called an $N$-ideal of $G$. An $N$-ideal $K$ of an $N$-module $G$ is called abelian, if $(K,+)$ is an abelian group and $(x+y) \alpha=x \alpha+y \alpha$ for all $x, y \in K$ and for all $\alpha \in N_{0}$ where $N_{0}$ is the zero-symmetric subnearring of $N$.

The following lemma is modified from [10, Proposition 2.1] and detailed proof will be included for easier reference.

Lemma 3.1. Let $N$ be a nearring, $G$ an $N$-module. Assume $H_{1}, H_{2}$ and $W$ are $\mathrm{N}$-ideals of $\mathrm{G}$ such that $\mathrm{G}$ is a direct sum of $\mathrm{N}$-ideals $\mathrm{H}_{1} \oplus \mathrm{H}_{2}$. If $\mathrm{H}_{2}$ is minimal and nonabelian, then either $W \subseteq H_{1}$ or $H_{2} \subseteq W$.

Proof. Assume on the contrary that $W \nsubseteq H_{1}$ and $H_{2} \nsubseteq \mathbb{W}$. Since $H_{1}$ is a maximal $N$-ideal of $G, H_{1}+W=G$. Notice that $k n=(k+0) n-0 n \in K$ for all $k$ in an arbitrary $N$-ideal $K$ and for all $n \in N_{0}$. It follows that any $N$-ideal $K$ is both an $N_{0}$-submodule and $N_{0}$-ideal of $G$. By Wielandt's Lemma [7, Lemma 3.22] and the minimality of $\mathrm{H}_{2}$, the $\mathrm{N}$-ideal

$$
\left[\left(H_{1}+W\right) \cap\left(H_{2}+W\right)\right] /\left[\left(H_{1} \cap H_{2}\right)+W\right] \simeq\left(H_{2}+W\right) / W \simeq H_{2}
$$

is abelian, contradicting the hypothesis that $\mathrm{H}_{2}$ is nonabelian. Hence result.

An $N$-module $G$ is called monogenic if there exists $g \in G$ such that $g N=G$. A monogenic $N$-module $G$ is said to be type- $O$ if $G$ has no nontrivial proper $N$ ideals. A monogenic $N$-module $G$ is called strongly monogenic if $g N=0$ or $g N=G$ for all $g \in G$. A strongly monogenic nontrivial $N$-module $G$ is said to be of type- 1 if $G$ has no nontrivial proper $N$-ideals. It is said to be of type-2 if $G$ has no nontrivial proper $N_{0}$-ideals. A nearring $N$ is called $\nu$-primitive on $G$ if $G$ is a faithful type- $\nu N$-module. A nearring which is not a ring is called a nonring.

Lemma 3.2. Let $Y_{1}, Y_{2}$ be 0 -closed subsets of a type-1 $N$-module $G$. If one of the following conditions holds, then $Y_{1} \cup Y_{2}$ is 0 -closed in $G$.

(1) $G$ is a nonabelian group.

(2) $N_{0} /(0: G)$ is a nonring.

Proof. Without lost of generality, we may suppose that both $Y_{1}$ and $Y_{2}$ are not empty. Let $U_{1}$ and $U_{2}$ be subsets of $N$ such that $Y_{1}=\left[0: U_{1}\right]$ and $Y_{2}=\left[0: U_{2}\right]$. Let $U=\left(0: Y_{1} \cup Y_{2}\right)$. From Proposition 2.4, we have

$$
Y_{1} \cup Y_{2} \subseteq\left[0:\left(0: Y_{1} \cup Y_{2}\right)\right]=[0: U] .
$$


Let $a \in[0: U]$. As $a \mu=0$ for all $\mu \in U$, we have $U \subseteq(0: a)$. If $\left(0: Y_{1}\right) \subseteq(0: a)$, then we have $a \in Y_{1}$ by Corollary 2.5. Thus $[0: U] \subseteq Y_{1}$. It follows that $[0: U]=Y_{1} \cup Y_{2}$ and $Y_{1} \cup Y_{2}$ is 0 -closed.

We may now assume that $\left(0: Y_{1}\right) \nsubseteq(0: a)$. Recall that $Y_{1}$ is not empty. If

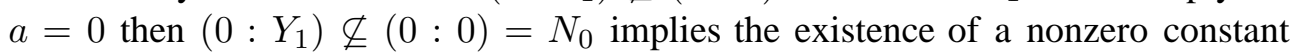
map $\theta_{c} \in N$ such that $Y_{1} \theta_{c}=0$, a contradiction. So $a \neq 0$. Since $G$ is strongly monogenic, $a N=0$ or $a N=G$. If $a N=0$ then $(0: a)=N$, consequently, $\left(0: Y_{1}\right) \subseteq(0: a)$, a contradiction. If $a N=G$ then $N /(0: a) \simeq G$ as a simple $N$-module. It follows that $(0: a)$ is a maximal right ideal of $N$, and so $N=(0: a)+\left(0: Y_{1}\right)$. Let $M=(0: a) \cap\left(0: Y_{1}\right)$. Then $N / M$ can be written as a direct sum as $N / M=(0: a) / M \oplus\left(0: Y_{1}\right) / M$, and so $\left(0: Y_{1}\right) / M$ is a minimal $N$-ideal of $N / M$. Now we have

$$
\left(0: Y_{1}\right) / M \simeq_{N}(N / M) /((0: a) / M) \simeq_{N} N /(0: a) \simeq_{N} a N=G .
$$

From the hypothesis, if $G$ is a nonabelian group, then clearly $\left(0: Y_{1}\right) / M$ is nonabelian. Note that $(0: G)$ is zero-symmetric and is an ideal in $N_{0}$. It is then routine to see that $G$ is a faithful $N_{0} /(0: G)$-module. If $N_{0} /(0: G)$ is a nonring, then from [7, Lemma 3.7.], we can conclude that $\left(0: Y_{1}\right) / M$ is nonabelian either. Hence $\left(0: Y_{1}\right) / M$ is a nonabelian minimal $N$-ideal of $N / M$.

By Lemma 3.1, we have either

$$
\left(0: Y_{1}\right) / M \subseteq\left(M+\left(0: Y_{2}\right)\right) / M
$$

or

$$
\left(M+\left(0: Y_{2}\right)\right) / M \subseteq(0: a) / M
$$

This implies that

$$
\text { either }\left(0: Y_{1}\right) \subseteq M+\left(0: Y_{2}\right) \text { or } M+\left(0: Y_{2}\right) \subseteq(0: a) \text {. }
$$

If $\left(0: Y_{1}\right) \subseteq M+\left(0: Y_{2}\right)=(0: a) \cap\left(0: Y_{1}\right)+\left(0: Y_{2}\right)$, then

$$
\left(0: Y_{1}\right)=(0: a) \cap\left(0: Y_{1}\right)+\left(0: Y_{2}\right) \cap\left(0: Y_{1}\right) .
$$

Moreover, $U=\left(0: Y_{1} \cup Y_{2}\right)=\left(0: Y_{1}\right) \cap\left(0: Y_{2}\right)$, and $U \subseteq(0: a)$, we thus have $\left(0: Y_{1}\right) \subseteq(0: a)$. It follows that $a \in Y_{1}$ by Corollary 2.5. On the other hand, if $M+\left(0: Y_{2}\right) \subseteq(0: a)$, it is clear that $\left(0: Y_{2}\right) \subseteq(0: a)$ or $a \in Y_{2}$ by Corollary 2.5. Therefore $[0: U] \subseteq Y_{1} \cup Y_{2}$. Hence $Y_{1} \cup Y_{2}=[0: U]$ is 0 -closed in $G$.

The following example shows that assuming $G$ is a type- $1 \mathrm{~N}$-module is not superfluous in Lemma 2.2. 
Example 3.3. There exists an $N$-module $G$ (not type-1) such that $G$ is a nonabelian group and $N=N_{0}$ is a nonring but the union of 0 -closed sets is not 0-closed.

Let $G=S_{3}=\{0, a, b, c, d, e\}$ be the symmetric group of degree $3, N=E\left(S_{3}\right)$ the nearring generated additively by the group endomorphisms of $S_{3}$. This nearring is of order 54 and a detailed description of all its elements is in [6, p. 70]. Consider $S_{3}$ as an $E\left(S_{3}\right)$-module. The alternating group $A_{3}=\{0, d, e\}$ is an $E\left(S_{3}\right)$-ideal of $S_{3}$. Thus the $E\left(S_{3}\right)$-module $S_{3}$ is not type-1. All the 0 -closed sets are $\emptyset,\{0\}$, $\{0, a\},\{0, b\},\{0, c\},\{0, d, e\},\{0, a, b, c\},\{0, a, d, e\},\{0, c, d, e\},\{0, b, d, e\}$ and $S_{3}$. The set $\{0, a, b\}=\{0, a\} \cup\{0, b\}$ is a union of 0 -closed sets but it is not 0 -closed.

The following corollary is a generalization of Scott's result [10, Theorem 2.2] on 2-primitive nearrings.

Corollary 3.4. Let $N$ be a 1-primitive nearring on a group $G$. If $G$ is a nonabelian group or $N_{0}$ is a nonring, then the union of 0-closed subsets in $G$ is o-closed.

Lemma 3.5. Let $G$ be an $N$-module and $K$ an $N$-ideal of $G$. Let $\rho: G \rightarrow$ $G / K$ be the canonical $N$-homomorphism. Then there is a one-one correspondence between the

Proof. Let $Y$ be a nonempty $K$-closed subset of $G$, and let $U \subseteq N$ such that $Y=[K: U]$. Clearly $Y \rho \subseteq\left[0_{G / K}: U\right]$. If $a+K \in\left[0_{G / K}: U\right]$, then $(a+K) \mu=0_{G / K}$ or $a \mu \in K$ for all $\mu \in U$. That is $a \in Y$ and $a+K \in Y \rho$. Hence $Y \rho=\left[0_{G / K}: U\right]$ and $Y \rho$ is 0 -closed in the $N$-module $G / K$. On the other hand, let $W$ be a nonempty 0 -closed subset of $G / K$ over $N$, say $W=\left[0_{G / K}: U\right]$ for some $U \subseteq N$. Claim that $W \rho^{-1}=[K: U]$. Since $K$ is an $N$-ideal, we have $(g+k) n-g n \in K$ for all $k \in K, g \in G, n \in N$.

Now let $a \in W \rho^{-1}$ and $\mu \in U$. From our assumption, we have

$$
a \mu+K=(a+K) \mu=(a \rho) \mu \in W U=0_{G / K} .
$$

Therefore $a \mu \in K$ and $a \in[K: U]$. Hence $W \rho^{-1} \subseteq[K: U]$.

If $a \in[K: U]$, then $a \mu \in K$ for all $\mu \in U$ and so we get $(a+K) \mu=a \mu+K=$ $K$ or $a \rho \in\left[0_{G / K}: U\right]=W$. Hence $a \in W \rho^{-1}$ and $[K: U] \subseteq W \rho^{-1}$.

Theorem 3.6. Let $G$ be an $N$-module and $K$ an $N$-ideal of $G$ such that $G / K$ is a type-1 $N$-module. If $G / K$ is a nonabelian group or $N_{0} /\left((K: G) \cap N_{0}\right)$ is a nonring, then the finite union of $K$-closed subsets of $N$-module $G$ are $K$-closed. 
Proof. This follows immediately from Lemma 3.2 and Lemma 3.5 and the fact that $\left(0_{G / K}: G / K\right)=(K: G)$.

From Corollary 2.2 and Theorem 3.6, a topology exists for a certain type-1 $N$-module $G$ with respect to a particular $N$-ideal $K$. This topology is referred as inverse $K$-topology in this paper. However, this topology is usually non-Hausdoff [ 8 , p. 98] as demonstrated in the following example.

Example 3.7. Let $G=\mathbb{Z}_{4}=\{0,1,2,3\}$ and $N=\left\{f \in M_{0}(G) \mid 3 f=0\right\}$. This group $G$ is a faithful type-2 $N$-module [7, Example 3.36]. The nearring $N$ is a 0 -symmetric nonring of order 16 . Hence the collection of all 0 -closed sets in $G$ forms a topology $\tau$. There are five 0 -closed sets in this topology, namely, $\mathbb{Z}_{4},\{0,1,3\},\{0,2,3\},\{0,3\}, \emptyset$. Therefore the collection of all open sets is $\tau=$ $\left\{\emptyset,\{2\},\{1\},\{1,2\}, \mathbb{Z}_{4}\right\}$. This topology is not Hausdoff for the singletons $\{0\}$ and $\{3\}$ can not be seperated by open sets.

Proposition 2.7 shows that result related to inverse quotient often has a dual one in noetherian quotient and vice versa. In this example, the collection of all sets in the form $(0: Y)$ for some $Y \subseteq G$ are: $N=(0: 0) ;\{(0000),(0010),(0020),(0030)\}=$ $(0: 1) ;\{(0000),(0100),(0200),(0300)\}=(0: 2) ;\{(0000)\}=(0:\{1,2\})$. Here the notation $(a b c d)$ represents the mapping in $N$ which maps $0,1,2,3$ to $a, b, c, d$ respectively. The union $(0: 1) \cup(0: 2)$ is a set of order 7 which cannot be the annihilator of any subsets of $G$. Therefore a possible dual for inverse 0-topology does not exist in this case.

The following result can be expected from the definition of $K$-closed set.

Theorem 3.8. Let $G$ be an $N$-module and $K$ an $N$-ideal of $G$ such that $G / K$ is a type-1 $N$-module. If $G / K$ is a nonabelian group or $N_{0} /\left((K: G) \cap N_{0}\right)$ is a nonring then the maps induced by the elements of $N$ are continuous from $G$ to $G$ with respect to

Proof. Let $Y$ be a $K$-closed subset of $G$ and $f \in N$. From Proposition 2.8, it follows that $Y f^{-1}=[Y: f]=[K: f(K: Y)]$ which is clearly $K$-closed in $G$ and so $f$ is continuous.

As indicated in Proposition 2.1(1), any $K$-closed set $[K: U]$ can be generated as the intersection of $K$-closed sets $[K: f]$ for all $f \in U$. In other words, arbitrary open sets in the inverse $K$-topology can be generated by union of open sets of the form $G \backslash[K: f]$ for $f \in N$. Explicitly,

$$
G \backslash[K: U]=G \backslash\left(\cap_{f \in U}[K: f]\right)=\cup_{f \in U}(G \backslash[K: f]) .
$$


Let $\beta_{K}\left(G_{N}\right)$ denote the set $\{G \backslash[K: f] \mid$ for all $f \in N\}$. Then $\beta_{K}\left(G_{N}\right)$ is indeed a base $[8$, p. 78] for the inverse $K$-topology in an $N$-module $G$. We write this as the following.

Proposition 3.9. Let $G$ be an $N$-module with inverse $K$-topology defined on $G$ where $K$ is an $N$-ideal of $G$. Let $\beta_{K}\left(G_{N}\right)=\{G \backslash[K: f] \mid$ for all $f \in N\}$. Then $\beta_{K}\left(G_{N}\right)$ is a base for this topology.

Proof. Assume $O$ is a nonempty open set in the inverse $K$-topology. Say $O=G \backslash[K: U]$ for some $U \subseteq N$. Since $O$ is nonempty, $U$ cannot be an empty set. Let $a \in O$ be arbitrary. Then $a \in G \backslash[K: f]$ for some $f \in U$. Let $C=G \backslash[K: f] \in \beta_{K}\left(G_{N}\right)$. Since $[K: U] \subseteq[K: f]$ by Corollary 2.3. It follows that $a \in C \subseteq O$. Thus $\beta_{K}\left(G_{N}\right)$ is a base for the inverse $K$-topology by [8, Lemma 13.2].

Proposition 3.9 will be helpful in finding examples to demonstate the inverse $K$-topology. In the following, examples will be given to motivate further stuty in this topic.

Example 3.10. (Near modules over nearring of polynomials) Let $G=\mathbb{R}$ be the group of real numbers, and $N=(\mathbb{R}[x],+, \circ)$ the nearring of polynomials using the usual addition of polynomials and substitution as multiplication $[1,2]$. Consider $\mathbb{R}$ as an $\mathbb{R}[x]$-module. The linear polynomial $\frac{b}{a} x \in \mathbb{R}[x]$ will map any nonzero $a$ to any chosen $b \in \mathbb{R}[x]$. Thus $\mathbb{R}$ is a type- $2 \mathbb{R}[x]$-module. The zero-symmetric part $\mathbb{R}_{0}[x]$ is a nonring, so the hypothesis in Theorem 3.6 hold.

Observe that $[0:-a+x]=\{a\}$ for all $a \in \mathbb{R}$. That is all singletons in $\mathbb{R}$ are 0 -closed. Thus the inverse 0 -topology is the finite complement topology [8, p. 77] on $\mathbb{R}$. Note that this is also the Zariski topology [3, p. 427] on $\mathbb{R}$.

Further, consider the reals $\mathbb{R}$ as an $\mathbb{R}_{0}[x]$-module. Using similar arguments as above, $\mathbb{R}$ is a type- $2 \mathbb{R}_{0}[x]$-module. Observe that $\left[0: a x-x^{2}\right]=\{0, a\}$ for all $a \in \mathbb{R}$. Thus all finite subsets containing $\{0\}$ in $\mathbb{R}$ are 0 -closed. In other words, the open sets in $\mathbb{R}$ are either $\mathbb{R}, \emptyset$ or those infinite subsets of $\mathbb{R}$ not containg $\{0\}$ and having finite complement.

Both topologies defined on $\mathbb{R}$ are $T_{1}$ but not $T_{2}$ and irreducible in the sense that any two nonempty open sets has nonempty intersection. In other words, the closure of any nonempty open set is $\mathbb{R}$.

Example 3.11. (Near modules over transformation nearrings) Let $G$ be a group with order at least 3 . Then the nearring of group mappings $M_{0}(G)=\{f: G \rightarrow G \mid$ $0 f=0\}$ is a nonring. Observe that the mapping $\alpha_{b}: G \rightarrow G$ such that $0 \alpha_{b}=0$ and $a \alpha_{b}=b$ for all $a \in G \backslash\{0\}$ is in $M_{0}(G)$. Thus $a M_{0}(G)=G$ for any nonzero $a \in G$. It follows that $G$ is both a type-2 $M(G)$-module and $M_{0}(G)$-module. For 
any nonempty set $C$ of $G$, define the mapping $\alpha_{C}: G \rightarrow G$ via $a \alpha_{C}=0$ for all $a \in C$ and $b \alpha_{C}=d$ for some fixed nonzero $d \in G$ and for all $b \in G \backslash C$. Then $\alpha_{C} \in M(G)$ and $\left[0: \alpha_{C}\right]=C$. Thus the inverse 0-topology in the $M(G)$-module $G$ is the discrete topology. Further, observe that $\alpha_{C} \in M_{0}(C)$ if and only if $0 \in C$. Thus the nonempty 0 -closed set in the $M_{0}(G)$-module $G$ is a subset containing 0 and vice versa. When $G$ is a simple nonabelian group, it is a type-2 $M_{c}(G)$-module. In this case, the inverse 0-topology is the trivial topology.

Proposition 3.9 shows that $\beta_{K}\left(G_{N}\right)$ is a base for the inverse $K$-topology. If the nearring $N$ is generated by a subsemigroup $S$ additively, we may ask: Is the set $\tau_{K}\left(G_{N}\right)=\{G \backslash[K: \alpha] \mid$ for all $\alpha \in S\}$ a subbase for this topology? This is not true in general. Let $G$ be a finite simple nonabelian group. Consider $G$ as an $M_{0}(G)$-module, Example 3.11 shows that the nonempty 0-open sets are those subsets not containing 0 . Observe that $M_{0}(G)=I(G)$ is generated additively by $\operatorname{Inn}(G)$, the group of inner automorphisms of $G$. But $[0: \alpha]=\{0\}$ for all $\alpha \in \operatorname{Inn}(G)$. Therefore $\tau_{0}\left(G_{M_{0}(G)}\right)=\{0\}$ which can not be the subbase for the inverse 0-topology.

Proposition 3.12. Let $N$ be a 0 -symmetric nearring with unity. Assume $G$ is a unital $N$-module with inverse 0-topology defined. Let $A$ be a nonempty 0 -closed subset of $G$. Then A contains no nonempty proper 0 -closed subset if and only if $(0: A)$ is a maximal right ideal of $N$.

Proof. Since $N$ contains unity, the right ideal $(0: A) \neq N$. Let $R$ be a right ideal of $N$ containing $(0: A)$. Then $[0: R] \subseteq[0:(0: A)]=A$ by Proposition 2.4. Observe that $0 \in[0: R]$ for $N$ is 0 -symmetric. Since $A$ contains no nonempty proper 0 -closed subset, $[0: R]=A$. It follows that $R \subseteq(0: A)$ and thus $(0: A)$ is maximal.

Conversely, if $A$ contains a nonempty proper 0 -closed subset $B$, then $(0: A) \subseteq$ $(0: B)$ by Corollary 2.5. If $(0: A)=(0: B)$, then $B=[0:(0: B)]=[0:(0:$ $A)]=A$ by Proposition 2.4 . Thus $(0: B)$ properly contains $(0: A)$. Since the $N$-module is unital, $1 \notin(0: B)$. Consequently, $(0: B)$ is a proper ideal of $N$ and $(0: A)$ can not be maximal. Hence result.

According to Proposition 3.12, we call a nonempty 0-closed set principle if it contains no nonempty proper 0 -closed subset. Recall that $a \in G$ is called a generator if $a N=G$. The following corollary provide a partial converse for Theorem 3.5.

Corollary 3.13. Suppose $N$ is a 0 -symmetric nearring with unity, $G$ a unital monogenic $N$-module with inverse 0-topology defined. Let $a \in G$ be a generator. Then the following are equivalent. 
(1) The doubleton $\{0, a\}$ is principlly closed.

(2) The annihilator $(0:\{0, a\})$ is a maximal right ideal of $N$.

(3) The annihilator $(0: a)$ is a maximal right ideal of $N$.

(4) The $N$-module $G$ is simple (i.e., $G$ has no nontrivial $N$-ideals).

(5) $G$ is a type-0 N-module.

Proof. The equivalence of (1) and (2) follows from Proposition 3.12. Further, it is clear that $(0:\{0, a\}) \subseteq(0: a)$. If $f \in(0: a)$, then $a f=0$ and $0 f=0$ for $N$ is 0 -symmetric. Thus $f \in(0:\{0, a\})$. Hence $(0:\{0, a\})=(0: a)$. This shows the equivalence of (2) and (3). Since $a$ is a generator, $G \simeq N /(0: a)$ as $N$-module. The equivalence of (3) and (4) follows immediately. For the last equivalence of (4) and (5), this follows the definition of type- $0 \mathrm{~N}$-module.

\section{REFERENCES}

1. G. F. Birkenmeier and F.-K. Huang, Annihilator conditions on polynomials, Comm. Algebra, 29 (2001), 2097-2112.

2. $\longrightarrow$, Annihilator conditions on polynomials II, Monatsh. Math., 141 (2004), 265276.

3. N. Jacobson, Basic Algebra II, W. H. Freeman, New York, 1989.

4. M. J. Johnson, Right ideals and right submodules of transformation near-rings, $J$. Algebra, 80 (1973), 386-391.

5. $\longrightarrow$ Maximal right ideals of transformation near-rings, J. Austral. Math. Soc., 19 (1975), 410-412.

6. J. J. Malone and C. G. Lyons, Endomorphism near-rings, Proc. Edinb. Math. Soc., 17 (1970), 71-78.

7. J. D. P. Meldrum, Near-rings and their links with groups, Pitman, London, Research Notes in Math., 134 (1985).

8. J. R. Munkres, Topology, 2nd. ed., Prentice Hall, New Jersey, 2000.

9. G. Pilz, Near-rings, 2nd. revised ed., North-Holland, Amsterdam, 1983.

10. S. D. Scott, Zero sets - consequences for primitive near-rings, Proc. Edinb. Math. Soc., 25 (1982), 55-63.

11. - Topology and primary $N$-groups, in: Near-rings and near-fields (Stellenbosch, 1997), Kluwer Acad. Publ., Dordrecht, 2001, pp. 151-197. 
Feng-Kuo Huang

Department of Mathematics,

National Taitung University,

Taitung 95002, Taiwan

E-mail: fkhuang@nttu.edu.tw 